\title{
Pen-Based Video Annotations: A Proposal and a Prototype for Tablet PCs
}

\author{
Diogo Cabral and Nuno Correia \\ CITI, Departamento de Informática, Faculdade de Ciências e Tecnologia, FCT, \\ Universidade Nova de Lisboa, Quinta da Torre, 2829-516 Caparica, Portugal \\ \{diogocabral, nmc\} @di.fct.unl.pt
}

\begin{abstract}
Pen computing or pen-based technology can be considered one of the most natural and intuitive computer input interface for humans. The combination of digital video and pen-based technology can support a new approach for electronic paper by associating handwritten notes and drawings with video segments. This paper presents a proposal for pen-based video annotations and a prototype for Tablet PCs, implementing this concept, particularly dynamic penbased video annotations combined with real-time video tracking.
\end{abstract}

Keywords: Annotations, Video Annotations, Pen-based Annotations, Penbased video Annotations, Tablet PCs.

\section{Introduction}

The pen is an old tool, which resulted from the need to communicate and memorize by writing down information [1]. The pen has changed through time as well as the substrate used to write [1]. The first attempts in pen computing occurred in the 1950s and 1960s [1] and the most recent and widely used development of this technology is the Tablet PC, a laptop computer equipped with pen-based technology and a touch screen. The size of the displays, almost the same as a regular sheet of paper, and its computational power, as a regular laptop, make the Tablet PCs an interesting approach to electronic paper and a precursor to interactions that will be possible in the future [2].

Annotations are a useful form of writing down information using pen and paper and can be associated to other media types like text and images, in a process that combines reading and critical thinking usually called active reading [3]. Nevertheless, annotations can also be applied to digital platforms and documents [3, 4]. More recently, digital video became a popular type of digital document and a medium of communication worldwide. Video can be considered one of the most complete and complex medium. Besides the pictorial and verbal (written text or speech) attributes, video includes motion and time characteristics. A process analogous to the active reading, called active watching, can associate annotations to video content [5].

The combination of digital video and pen-based technology allows association between handwritten annotations/drawings with video segments. Pen-based video annotations introduce new notions for the note taking act, such as temporal position and motion, when compared with regular pen and paper annotations. 


\section{Related Work}

The topic of pen-based video annotations is not new and some systems $[6,7,8,9,10]$ already explored this idea. Nevertheless, most of them $[6,8,9,10]$ do not totally explore the dynamic dimension of video content, making the annotations spatially static, while the content is moving. Although, the Ambulant Annotator [7] explores this dimension, the annotation spatial path is defined by the user and not by tracking the video content. A more recent work [11] explores graphical video annotations combined with motion tracking, but do not consider the particular case of pen-based annotations and the tracking feature needs a long time of video pre-processing.

\section{Pen-Based Video Annotations}

The input modality of digital annotations can be divided in three types: ink, keystroke and multimedia [12]. Pen-based annotations generate ink-based annotations; nevertheless, if a keyboard annotation is a set of characters [12], then a pen-based annotation can be considered a set of ink strokes. Each ink stroke has its own attributes, like color or thickness, which can differ from one to another [3]. Therefore, a pen-based annotation can be considered a set of ink strokes with different attributes. In addition, a temporal dimension can be added to the ink strokes. Normally, a user makes these ink strokes sequentially [4]. This temporal order associated to each ink stroke can be crucial to understand the idea transmitted by the annotation.

Video annotations should include spatial and temporal dimensions also associated with video content [5]. Together, these dimensions generate the idea of motion, spatial position that varies with time, which should be also associated to video annotations. Video annotations should have the ability to follow specific motion features included in the video content.

The combination of both concepts, pen-based annotations and video annotations, generate a new kind of annotations, pen-based video annotations, which can be defined as annotations composed by a set of time dependent ink strokes, each with specific attributes, and associated with video changeable temporal and spatial positions.

The attributes of pen-based video annotations can include ink properties (e.g. color, thickness), private or public definitions, hypertext properties (e.g. spatial anchors on the video content and links to other media types) and tracking features (e.g. tracking objects, colors and textures).

\section{Prototype}

A prototype was developed with the aim to implement pen-based video annotations. The system was developed for Tablet PCs (Fig. 1a) and includes pen-based annotations displayed over video content, associated by time to video intervals, and with some changeable ink attributes, such as color and thickness. In addition, the system has a frame based timeline making possible to browse the video content, by selecting 


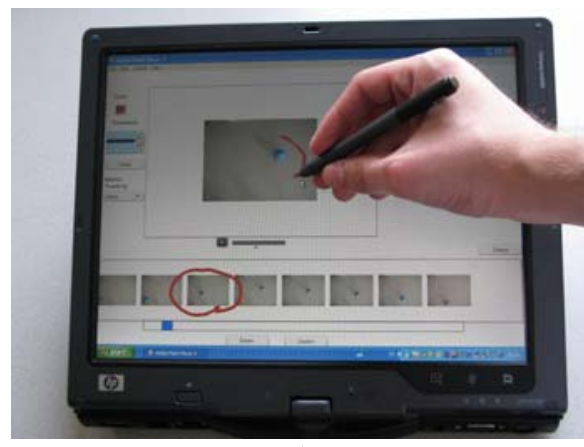

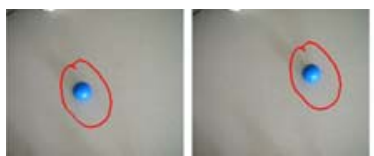

b)

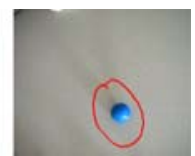

a)

Fig. 1. a) The prototype running on a Tablet PC b) Annotation Motion Tracking

a particular frame, and to draw over a set of frames. A scrollbar was also developed, with the aim to navigate through the frame timeline, with the particular feature that the marker has fixed dimensions, sufficient to be selected using the pen, without missing the target, as can happen with regular scrollbars.

The prototype includes annotations motion tracking, which allows annotations to follow motion changes in the video content, as shown in Fig. 1b.

The motion tracking is based on the difference of frames on the area defined by the annotation, in real-time. The annotation area, defined by a rectangle embracing the ink strokes, is equally divided in four smaller rectangles and the rectangle (of these four) that presents a larger change of pixels, represents the direction that the annotation should follow. This method does not require a lot of processing power, enabling its real-time usage. The annotations are drawn for every frame in a transparent layer over the video window, without the need to change the original video content. The system also redraws the ink strokes in the same order that they were made. The annotation lifetime is by default equal to the video time length but the time interval can be changed using a dual slider. Annotation motion stops when the object stops or when it is not visible in the video window.

\section{Conclusions and Future Work}

This paper presents a proposal for pen-based video annotations and the different notions introduced by this type of annotations. The dynamic property of pen-based annotations was implemented in a prototype using Tablet PCs, allowing an annotation to track video content movements, among other features. Additional capabilities, such as pressure and gesture widgets and tracking other features and multiple objects will be developed as next steps. Finally, usability tests will be carried out to test the proposed system.

Acknowledgments. The presented work is partially funded by the UTAustin-Portugal Program and by the HP Technology for Teaching Grant Initiative 2006. 


\section{References}

1. Meyer, A.: Pen Computing, A Technology Overview and a Vision. SIGCHI Bulletin 27, 46-90 (1995)

2. Hayes, R.A., Feenstra, B.J.: Video-speed electronic paper based on electrowetting. Nature 425, 383-385 (2003)

3. Marshall, C.C.: Annotation: from paper books to the digital library. In: 2nd ACM International Conference on Digital libraries, pp. 131-140. ACM Press, New York (1997)

4. Bargeron, D., Moscovich, T.: Reflowing digital ink annotations. In: SIGCHI Conference on Human factors in Computing Systems CHI 2003, pp. 385-393. ACM Press, New York (2003)

5. Correia, N., Chambel, T.: Active video watching using annotation. In: 7th ACM International Conference on Multimedia (Part 2), pp. 151-154. ACM Press, New York (1999)

6. Ramos, G., Balakrishnan, R.: Fluid interaction techniques for the control and annotation of digital video. In: 16th annual ACM Symposium on User interface Software and Technology, pp. 105-114. ACM Press, New York (2003)

7. Bulterman, D.C.A.: Animating Peer-Level Annotations Within Web-Based Multimedia. In: Eurographics Multimedia 2004, pp. 49-57. Eurographics Association (2004)

8. Diakopoulos, N., Essa, I.A.: Videotater: an approach for pen-based digital video segmentation and tagging. In: 19th annual ACM symposium on User interface software and technology, pp. 221-224. ACM Press, New York (2006)

9. Rahn, N.C., Lim, Y., Groth, D.P.: Redesigning video analysis: an interactive ink annotation tool. In: CHI 2008 Extended Abstracts on Human Factors in Computing Systems, pp. 3339-3344. ACM Press, New York (2008)

10. Graça, C., Pimentel, M., Goularte, R., Cattelan, R.G., Santos, F.S., Teixeira, C.: Ubiquitous Interactive Video Editing Via Multimodal Annotations. In: Tscheligi, M., Obrist, M., Lugmayr, A. (eds.) EuroITV 2008. LNCS, vol. 5066, pp. 72-81. Springer, Heidelberg (2008)

11. Goldman, D.B., Gonterman, C., Curless, B., Salesin, D., Seitz, S.M.: Video object annotation, navigation, and composition. In: 21th annual ACM Symposium on User Interface Software and Technology, pp. 3-12. ACM Press, New York (2008)

12. Cousins, S.B., Baldonado, M., Paepcke, A.: A Systems View of Annotations. Technical Report, Xerox PARC (2000) 